Published in final edited form as:

Pain. 2007 September ; 131(1-2): 83-95.

\title{
Neuropeptide $\mathrm{Y}$ acts at $\mathrm{Y} 1$ receptors in the Rostral Ventral Medulla To Inhibit Neuropathic Pain
}

\author{
Bradley K. Taylor ${ }^{1,}{ }^{*}$, Sarang S. Abhyankar ${ }^{2}$, Ngoc-Tram T. Vo ${ }^{1}$, Christopher L. Kriedt ${ }^{1}$, Sajay \\ B. Churi ${ }^{1}$, and Janice H. Urban ${ }^{3}$ \\ 1 Department of Pharmacology, School of Medicine, Tulane University, New Orleans, LA 70112, USA \\ 2 School of Pharmacy, University of Missouri-Kansas City \\ 3 Department of Physiology and Biophysics, Rosalind Franklin University of Medicine and Science, North \\ Chicago, IL
}

\begin{abstract}
Brain microinjection studies in the rat using local anesthetics suggest that the rostral ventral medulla (RVM) contributes to the facilitation of neuropathic pain. However, these studies were restricted to a single model of neuropathic pain (the spinal nerve ligation model) and to just two stimulus modalities (non-noxious tactile stimulus and heat). Also, few neurotransmitter systems have been shown to modulate descending facilitation. After either partial sciatic nerve ligation (PSNL) or spared nerve injury (SNI), we found that unilateral or bilateral microinjection of lidocaine into the RVM reduced not only mechanical allodynia (decreased threshold to von Frey hairs and/or an automated device) and mechanical hyperalgesia (increased paw lifting in response to a noxious pin), but also cold hypersensitivity (increased lifting in response to the hindpaw application of a drop of acetone). Application of a drop of water did not elicit paw withdrawal, indicating that the acetone test is indeed a measure of cold hypersensitivity. We found significant neuropeptide Y Y1-like immunoreactivity within, and lateral to, the midline RVM. Intra-RVM injection of neuropeptide Y (NPY) dosedependently inhibited the mechanical and cold hypersensitivity associated with PSNL or SNI, an effect that could be blocked by the Y1 receptor antagonist BIBO 3304. We conclude that medullary facilitation spans multiple behavioral signs of allodynia and hyperalgesia in multiple models of neuropathic pain. Furthermore, NPY inhibits behavioural signs of neuropathic pain, possibly by acting at $\mathrm{Y} 1$ receptors in the RVM.
\end{abstract}

\section{Keywords}

descending facilitation; allodynia; hyperalgesia; spared nerve injury; lidocaine; rat

\section{INTRODUCTION}

The brain exerts a powerful influence on the spinal transmission of pain signals. Medullary pathways have received the most attention, particularly those arising from the rostral ventral medulla (RVM). Substantial evidence indicates that the RVM contributes to both facilitation and inhibition of acute and persistent pain (Porreca et al. 2002;Ren and Dubner 2002). For

\footnotetext{
*Corresponding author: Bradley K Taylor, Department Pharmacology, SL83, Tulane University Health Sciences Center, New Orleans, LA 70112 USA, Tel: 504988 3354, Fax: 504988 5283, E-mail address: taylorb@ tulane.edu, http://www.som.tulane.edu/faculty/taylorb/ index.htm

Publisher's Disclaimer: This is a PDF file of an unedited manuscript that has been accepted for publication. As a service to our customers we are providing this early version of the manuscript. The manuscript will undergo copyediting, typesetting, and review of the resulting proof before it is published in its final citable form. Please note that during the production process errors may be discovered which could affect the content, and all legal disclaimers that apply to the journal pertain.
} 
example, treatments which inhibit the activity of RVM neurons, such as focal lesions, local anaesthetics, or transection of descending funicular tracts, substantially reduce tactile and/or heat hypersensitivity (Pertovaara et al. 1996; Miki et al. 2002;Robinson et al. 2002; Chen et al. 2004). These studies were restricted to a single model of neuropathic pain (the spinal nerve ligation model) and a single measure of either allodynia (von Frey hairs) and/or hyperalgesia (heat). However, the magnitude of behavioral responses to specific stimulus modalities, and their sensitivity to analgesic drugs, differs widely among neuropathic pain models (Kim et al. 1997; Chen et al. 2004;Decosterd et al. 2004;Erichsen et al. 2005;Pertovaara et al. 2005).

Therefore, our first goal was to extend a key finding in the spinal nerve ligation model, that local anesthetic-induced inhibition of medullary neural activity reduces mechanical allodynia in the von Frey test, to two additional models of neuropathic pain using multiple sensory modalities. We evaluated the effects of intra-RVM lidocaine following either partial ligation of the sciatic nerve (Seltzer et al. 1990) or section of the tibial and common peroneal branches of the sciatic nerve, sparing the sural branch (Decosterd and Woolf 2000). We evaluated tactile sensitivity not only with von Frey hairs, but also with machine-mounted filaments. We measured noxious mechanical and cold sensitivity at the plantar hindpaw in response to application of a noxious pin or a drop of acetone, respectively.

While numerous neurotransmitters have been discovered to modulate the outflow of descending inhibitory neurons at the level of the RVM, such as GABA, opioid peptides, serotonin, excitatory amino acids, neurotensin, and norepinephrine (Fields et al. 1991), only a handful have been suggested to modulate the descending facilitation of neuropathic pain, such as opioid peptides, cholecystokinin, neurotensin, and glutamate (Porreca et al. 2002). NPY Y1 receptors are an interesting target because they are found in the midline raphe magnus (Kopp et al. 2002), a key pain modulatory center in the RVM. Furthermore, microinjection of NPY into the RVM inhibits acute nociception in the hotplate test, an effect that could be reversed with NPY (28-36) (Zhang et al. 2000). However, no studies have evaluated the behavioural effects of NPY in a model of chronic pain. Therefore, to test the hypothesis that NPY attenuates behavioural signs of neuropathic pain, we evaluated the anti-allodynic and anti-hyperalgesic effects of microinjection of NPY, with or without NPY receptor antagonists, into the RVM.

\section{MATERIALS AND METHODS}

\section{Animals}

For the behavioral pharmacology studies, male Sprague-Dawley rats (Charles Rivers Laboratories, Inc, Portage) were 230-240g at time of nerve injury, 270-290g at time of stereotaxic surgery, and 320-370g during pharmacological testing. Animals were housed in individual cages on a 12-hour light/dark cycle starting at 6 a.m., and were given food and water ad libitum. All animal use protocols were approved by the institutional animal care and use committee (IACUC) of both UMKC and Tulane University. For the Y1 immunohistochemistry studies, male Sprague-Dawley rats (Charles River; 250-350g) were maintained under standardized lighting conditions (14:10 light:dark cycle) in a temperature-controlled environment $\left(23^{\circ} \mathrm{C}\right)$ with free access to rat chow and tap water. Procedures were approved by the Rosalind Franklin University of Medicine and Science (IACUC).

\section{Nerve injury surgery}

Rats were anesthetized with isoflurane (5\% induction, then $1.5 \%$ maintenance in oxygen). The partial sciatic nerve ligation (PSNL) studies were performed at the University of MissouriKansas City. As previously described (Seltzer et al. 1990), an incision was made at the skin overlying the lateral femur, exposing the sciatic nerve. The nerve was dissected from surrounding connective tissue near the trocanter, just distal to the branching point of the posterior biceps semitendinosus nerve. A tight ligature was tied around to $1 / 2$ the diameter of 
the nerve with 9-0 silk suture. The spared nerve injury (SNI) studies were performed at Tulane University. As described previously (Decosterd and Woolf 2000), an incision was made in the skin at the level of the trifurcation of the left sciatic nerve. The overlying muscles were retracted, exposing the common peroneal, tibial, and sural nerves. The common peroneal and tibial nerves were ligated with 6.0 silk (Ethicon, Somerville, NJ) and then the knot and adjacent nerve $(2 \mathrm{~mm})$ were transected. Care was taken to avoid touching the sural branch. Sham surgery was produced by skin incision at the level of the trifurcation, and by exposing but not touching the three sciatic branches. Following PSNL, SNI, or sham surgery, the muscle was sutured with absorbable 4.0 sutures (Ethicon) and the wound was closed with metal clips.

\section{Stereotaxic implantation of microinjection cannulae}

Three to five days after nerve injury, animals were re-anesthetized with isoflurane, and then placed in a stereotaxic apparatus (Stoelting). Following an incision to expose the cranium, the dorsal surface of the skull was levelled at lambda and bregma. Except for the lidocaine study in SNI rats, cannulae (17 mm, 23G, plugged with a 30G wire stylet) were placed above the RVM using interaural coordinates AP - 1.9mm; ML - 0.0, DV - $0.5 \mathrm{~mm}$. Cannulae were secured with three machine screws and dental cement (Lang Dental, Wheeling, IL). After closing the wound with 6.0 silk sutures, rats were housed singly for an additional recovery period of 6- $8 \mathrm{~d}$ before testing. The SNI lidocaine studies were performed similarly, except that $26 \mathrm{G}$ cannulae (with a 33G wire stylet; Plastics One, Roanoke, VA) were implanted bilaterally using stereotaxic mediolateral coordinates of 0.6.

\section{Drug Administration}

Drugs were administered via remote microinjection to minimize effects of animal handling. PE-10 tubing, filled with saline or drug, was used to connect a 1- $\mu$ Hamilton microsyringe to a 30G microinjector. The rat was removed from its testing box, and the stylet was replaced with the microinjector, which extended $1 \mathrm{~mm}$ beyond the tip of the guide cannula. The animal was returned to its testing box. After an additional $10 \mathrm{~min}$ of acclimation, $0.5 \mu \mathrm{l}$ of saline, lidocaine (2-4\%) or NPY (0.3-30 $\mu \mathrm{g})$ was slowly infused over $1 \mathrm{~min}$. Progress of the injection was visually confirmed by observation of movement of a small air bubble within the PE-10. Microinjectors were left in place an additional minute to minimize backflow along the cannula tract.

\section{Behavioral tests of allodynia and hyperalgesia}

Animals were acclimated to a stainless steel grid within individual Plexiglas boxes for 30-60 min, and then tested for mechanical allodynia, mechanical hyperalgesia, and/or cold hypersensitivity. Mechanical allodynia was assessed first with von Frey monofilaments (Stoelting), and/or a machine-mounted filament (Dynamic Plantar Anesthesiometer, Ugo Basile), and then mechanical hyperalgesia and/or cold hypersensitivity were assessed with a blunt pin and/or acetone, as further described.

Von Frey assessment of tactile threshold-In all animals, we first assessed mechanical allodynia using von Frey (Stoelting, Inc). The plantar region of each hind paw was stimulated with an incremental series of 8 monofilaments of logarithmic stiffness. In SNI rats, we further localized the stimulus region to the sural innervation territory of the lateral aspect of the plantar hind paw. The 50\% withdrawal threshold was determined using the up-down method of Dixon, modified by Chaplan et al (Chaplan et al. 1994). First, an intermediate von Frey monofilament (number 4.31, exerts $2.0 \mathrm{~g}$ of force) was applied perpendicular to the plantar skin, causing a slight bending. In case of a positive response (rapid withdrawal of the paw within $6 \mathrm{sec}$ ), a smaller filament was tested. In case of a negative response, a larger filament was tested. Some SNI animals $(\sim 15 \%)$ did not develop mechanical allodynia on the day of pharmacological 
testing after nerve injury (VF threshold $>5.0 \mathrm{~g}$ on the nerve-injured side). In such cases, von Frey testing was either terminated or its data was not included in the final analysis.

Automated assessement of tactile threshold-In the SNI studies, mechanical allodynia was also assessed with machine-mounted filaments (Ugo Basile, Inc). Under visual guidance with an attached angled mirror, the thin metal blunt rod of this device was positioned under the lateral region of the plantar hindpaw. A trigger was depressed, initiating contact of the rod to the footpad. The applied force increased at a rate of $2.5 \mathrm{~g} / \mathrm{sec}$ with a cut-off of $50 \mathrm{~g}$ at $20 \mathrm{sec}$. Upon paw withdrawal, the device automatically disengaged and printed out the applied force. At least 3, and usually $4-5$, measurements were averaged. Some SNI animals $(\sim 10 \%)$ did not develop mechanical allodynia on the day of pharmacological testing after nerve injury (percentage decrease after SNI $<25 \%$ ). In such cases, machine-mounted filament testing was either terminated or its data was not included in the final analysis.

Test of mechanical hyperalgesia-Next, we gently applied the sharp edge of a dull pin to the footpad, avoiding damage to the skin. The duration of paw withdrawal was recorded for either $10 \mathrm{sec}$ or 30 seconds in PSNL or SNI rats, respectively. Three observations were averaged. Because many SNI animals ( $>30 \%$ ) did not develop mechanical hyperalgesia on the day of pharmacological testing after nerve injury (response duration $<2 \mathrm{sec}$ ), we concluded that this form of mechanical hyperalgesia is not reliable in the SNI model in the setting of intracranial cannulation. In the absence of cannulation, other studies indicate a much more robust paw withdrawal in response to application of a pin to the footpad, indicative of reliable mechanical hyperalgesia in the SNI model (Taylor et al, unpublished observations, and Decosterd and Woolf, 2000).

Test of cold allodynia-Using a syringe connected to PE-90 tubing, flared at the tip to a diameter of $3 \frac{1}{2} \mathrm{~mm}$, we applied a drop of acetone (or water) to the plantar paw. Surface tension maintained the volume of the drop to 10-12 $\mu \mathrm{l}$. The length of time the animal lifted or shook its paw was recorded. The duration of paw withdrawal was recorded for either $10 \mathrm{sec}$ or 30 seconds in PSNL or SNI rats, respectively. Three observations were averaged. Some SNI animals $(\sim 20 \%)$ did not develop cold hypersensitivity on the day of pharmacological testing after nerve injury (response duration $<2 \mathrm{sec}$ ). In such cases, acetone testing was either terminated or its data was not included in the final analysis. In the final study, we confirmed cold allodynia by placing the animals in a $1 \mathrm{~cm}$ deep ice bath, followed by observation of lifting behaviour of the ipsilateral paw for $60 \mathrm{~s}$.

\section{Histological verification of injection site}

Under deep pentobarbital anesthesia (100-200 mg/kg), $0.5 \mu \mathrm{L}$ India ink was injected with a microinjector. Rats were then transcardially perfused with PBS (100-150 ml) and then $10 \%$ formalin (200-300 ml). An additional $0.5 \mu \mathrm{L}$ India ink was injected. The brains were removed, cryoprotected in $30 \%$ sucrose for at least $48 \mathrm{hr}$, sectioned at $50 \mu \mathrm{m}$ on a freezing microtome, briefly placed in PBS and then every fourth section was immediately mounted on slides to minimize leaching of the ink. After drying, sections were counterstained with cresyl violet and then cover-slipped. Without knowledge of the experimental results, we mapped the location of the microinjection cannula. We only used results from animals where the cannulae were centered near the ventromedial medulla at the rostral-caudal level of the facial nucleus, as illustrated in our previous studies (Taylor and Basbaum 1995;2003) and in Figs 1A-B.

\section{Immunohistochemistry}

Animals were anesthetized with an intraperitoneal (i.p.) injection of sodium pentobarbital (Anpro Pharmaceutical; $50 \mathrm{mg} / \mathrm{kg}$ ) and transcardially perfused with $25 \mathrm{ml}$ of phosphatebuffered saline (PBS; $10 \mathrm{mM} \mathrm{Na}_{2} \mathrm{HPO}_{4}, 150 \mathrm{mM} \mathrm{NaCl}, \mathrm{pH} 7.5$ ) containing $0.1 \%$ procaine and 
$100 \mathrm{U} / \mathrm{ml}$ heparin at $37^{\circ} \mathrm{C}$ followed by $50 \mathrm{ml}$ fixative solution [4\% paraformaldehyde in PBS (pH 8.0)] at $+4^{\circ} \mathrm{C}$. Brains were rapidly dissected out, post-fixed for 18-24 hours in fixative solution at $+4^{\circ} \mathrm{C}$ and stored in PBS (pH 7.5) at $+4^{\circ} \mathrm{C}$. Coronal brain sections were cut at 40 $\mu \mathrm{m}$ thicknesses on a vibratome and free-floating sections were collected in ice-cold PBS (pH 7.5) and processed for immunohistochemical staining.

Immunohistochemical procedures have been described previously (Wolak et al. 2003). Freefloating sections were washed in ICC buffer [0.2\% gelatin, $0.01 \%$ thimerosal, $0.002 \%$ neomycin in PBS (pH 7.5)]. The tissue was blocked with $2 \%$ normal goat serum (NGS) for 30 minutes at $+4^{\circ} \mathrm{C}$ and then incubated for 48 hours at $+4^{\circ} \mathrm{C}$ with either the rabbit anti-Y1 receptor antiserum $(1: 2,000)$ in ICC buffer with $2 \%$ NGS. Sections were washed in ICC buffer and incubated for 45 minutes at room temperature with biotinylated, affinity purified goat antirabbit IgG, followed by subsequent washes in ICC buffer and avidin-biotin-horseradish peroxidase complex (VECTASTAIN Elite ABC, Vector Laboratories; 1:300; $45 \mathrm{~min}$ ). Sections were washed in Tris-buffered saline (TBS; $100 \mathrm{mM}$ Tris base, $150 \mathrm{mM} \mathrm{NaCl}, \mathrm{pH} 7.5$ ) and signal was developed by incubating sections with $0.03 \%$ 3-3' diaminobenzidine (DAB), $0.01 \% \mathrm{H}_{2} \mathrm{O}_{2}$ and $0.03 \% \mathrm{NiCl}_{2}$ in TBS ( $\mathrm{pH}$ 7.5). The signal developed for 15-20 minutes, and the sections were washed in TBS and mounted on gelatin-coated slides. Sections were finally dehydrated in a series of ascending ethanol washes $(70,80,90,100 \%)$ and cleared in a xylene substitute (Hemo-De; Fisher). Coverslips were applied with Permount. Brain sections through the brainstem were atlas matched using a stereotaxic rat atlas (Paxinos and Watson 1997) and immunoreactive staining was visualized by light microscopy. Signal specificity was assessed by preadsorbing the antibody with 100 -fold excess of the Y1 receptor peptide sequence used to generate the antibody. Images were captured using a SPOTII digital camera and MetaMorph software. The contrast and brightness of the micrographs were adjusted, and montages were assembled, using Adobe Photoshop.

\section{Materials}

Human NPY, obtained from Anaspec (San Jose, CA), was diluted in saline, divided into aliquots, and frozen at $-70^{\circ} \mathrm{C}$ until use. All other drugs were prepared fresh daily. The Y1 receptor antagonist BIBO 3304 was generously provided by H. Doods (Boehringer Ingelheim, Biberach, Germany). Lidocaine was obtained from Henry Schein. Saline was obtained from Baxter (Deerfield, IL). Isoflurane was from Abbott Labs (Chicago, Ill).

\section{Data Analysis}

Using Systat 11 software, differences between means of parametric data (MMF, mechanical hyperalgesia, cold hypersensitivity) were analyzed by two-way repeated-measures ANOVA. Drug treatment was the between-subjects factor and Time was the repeated measure. If significant $(\mathrm{P}<0.05)$, the analyses were followed by post-hoc $\mathrm{t}$-tests with Bonferroni correction to evaluate group differences at specific time-points. Differences between nonparametric data (von Frey hairs) were analyzed by Kruskal-Wallis (\# groups>2) and/or Mann-Whitney (\# groups $=2$ ) statistics. Data are presented as mean \pm S.E.M.

\section{RESULTS}

\section{Y1 immunohistochemistry in the RVM}

Examination of atlas matched brain sections in the rostral medulla indicate that $\mathrm{Y} 1$ receptor immunoreactivity is present on cells scattered throughout the RVM, particularly around those areas corresponding to the site of cannulae placement (Figs 1C and 1D). Higher magnification (Figs $1 \mathrm{E}$ and $1 \mathrm{~F}$ ) demonstrates the presence of $\mathrm{Y} 1$ receptor immunoreactivity on scattered cell bodies and fibers in regions that are located at, and lateral to, the midline. Morphologically, these Y1 receptor immunopositive cell bodies are between 15-20 $\mu \mathrm{m}$ in diameter and exhibit 
either a fusiform or multipolar shape. In general, cells demonstrate moderate labelling for the Y1 receptor on the cell body and proximal processes. Numerous lightly stained NPY Y1 receptor-immunopositive fibers are also scattered throughout the rostral medulla.

\section{SNI model: RVM Lidocaine}

Consistent with previous publications, we used a bilateral microinjection approach to deliver lidocaine to the RVM (Pertovaara et al. 1996; Chen et al. 2004). As illustrated in Fig 2A-C, spared nerve injury produced robust mechanical allodynia as assessed with a drop in threshold to either von Frey (VF) or machine-mounted filaments (MMF). SNI also produced cold allodynia, as assessed by robust withdrawal responses in the acetone test. We found that bilateral intra-RVM delivery of $2 \%$ lidocaine increased $\mathrm{VF}\left[\mathrm{X}^{2}=6.7, \mathrm{P}<0.01\right]$ and MMF threshold $[\mathrm{F}(1,24)=6.4, \mathrm{P}<0.05]$ and decreased cold allodynia $[\mathrm{F}(1,21)=10.3, \mathrm{P}<0.005]$. As expected for a short-acting local anesthetic, this response was largely over within $45 \mathrm{~min}$. Lidocaine did not change behavioral responses evoked from the contralateral paw $(\mathrm{P}>0.05)$.

For consistency with the NPY microinjection studies (below), we also tested the antiallodynic effect of unilateral microinjection of lidocaine into the midline RVM. As illustrated in Fig 2D$\mathrm{F}$, unilateral intra-RVM delivery of $4 \%$ lidocaine increased VF but not MMF threshold, and decreased cold allodynia. Lidocaine did not change behavioral responses evoked from the contralateral paw (not shown).

\section{SNI model: RVM NPY}

Intra-RVM NPY inhibits transient nociception (Zhang et al. 2000). Fig 3 extends this finding to the SNI model of neuropathic pain. NPY dose-dependently and reversibly inhibited behavioural signs of mechanical allodynia on the ipsilateral side as assessed with either von Frey hairs (at $\mathrm{t}=15$, for example, Kruskal-Wallis $(\mathrm{df}, 3)=10.1, \mathrm{P}<0.05)$ or a machine-mounted filament $[\mathrm{F}(3,25)=6.6, \mathrm{P}<0.005$ by repeated-measures ANOVA, $\mathrm{t}=15-120]$, and also inhibited cold allodynia $[\mathrm{F}(3,23)=9.4, \mathrm{P}<0.001$ by repeated-measures ANOVA, $\mathrm{t}=15-120]$. NPY did not change behavioral responses evoked from the contralateral paw (although close inspection of Fig 3D suggests that NPY increased mechanical threshold, this did not reach statistical significance $[\mathrm{P}>0.05])$.

\section{PSNL model: RVM Lidocaine}

We next evaluated the effect of intra-RVM lidocaine in an alternative model of neuropathic pain, involving partial ligation of the sciatic nerve (PSNL). In this model we used only the von Frey test to evaluate mechanical allodynia, and Fig 4A illustrates that this was reduced by lidocaine [at $\mathrm{t}=60$, for example, Mann-Whitney $(\mathrm{df}, 1)=9.1, \mathrm{P}<0.005$ ]. Lidocaine also reduced behavioural signs of mechanical hyperalgesia $[\mathrm{F}(3,96)=25.19 ; \mathrm{P}<0.0001]$ and the duration of the withdrawal response to acetone $[\mathrm{F}(3,100)=12.44 ; \mathrm{P}<0.0001]$ at the ipsilateral side. Lidocaine did not alter responses at the contralateral side [P>0.05].

\section{PSNL model: RVM NPY}

We next confirmed the anti-allodynic effects of intra-RVM administration of NPY in the PSNL model, and extended these findings to a behavioural test of mechanical hyperalgesia. As illustrated in Figure 5, NPY clearly reduced mechanical allodynia [at $\mathrm{t}=10$, for example, MannWhitney $(\mathrm{df}, 1)=15.3, \mathrm{P}<0.001\}$ and mechanical hyperalgesia $[\mathrm{F}(1,47)=27, \mathrm{P}<0.001\}$ and also reduced the duration of paw withdrawal to acetone $[\mathrm{F}(1,47)=27, \mathrm{P}<0.001]$ at the ipsilateral side. NPY did not alter responses on the contralateral side $(\mathrm{P}>0.05)$.

To determine the effect of NPY dose on behavioural signs of neuropathic pain, we compared the effects of intra-RVM saline with 3 doses of NPY, including a repetition of the $1 \mu \mathrm{g}$ dose. 
We did not further evaluate acetone responses in this study due to the high ratio of variability/ response magnitude (SEM/response $\sim 1 \mathrm{~s} / 3 \mathrm{~s}=33 \%$ ) associated with the acetone test in the PSNL model (see Fig 5E-F). Indeed, the weak magnitude of the response to acetone (much shorter than the response to cold water or acetone in the SNI model) raises questions as to whether cold allodynia is reliably produced in the PSNL model. As illustrated in Fig 6, NPY dose-dependently decreased mechanical allodynia [at $\mathrm{t}=10$, for example, Kruskal-Wallis (df, 3 ) $=14.9, \mathrm{P}<0.005]$ and mechanical hyperalgesia at the ipsilateral side. NPY did not alter responses on the contralateral side $(\mathrm{P}>0.05)$.

\section{PSNL model: RVM NPY Y1 receptor antagonist}

To test the hypothesis that the Y1 receptor mediates the antiallodynic and antihyperalgesic effects of NPY, we administered the selective Y1 receptor antagonist, BIBO3304. As illustrated in Fig. 7, BIBO3304 blocked the effects of NPY. However, when administered by itself, BIBO3304 had no significant effect $(\mathrm{P}>0.05)$.

\section{The acetone test is a measure of cold hypersensitivity in the SNI model}

When applied to the paw, a drop of acetone stimulates both thermal and mechanical receptors in the plantar skin. We compared paw withdrawal responses to a drop of acetone, a drop of water, or ice-cold water after SNI. As illustrated in Figs 8A-B, a drop of water did not mimic the effect of acetone. In contrast, as illustrated in Fig 8C, ice water produced a robust paw withdrawal response that lasted as long as the acetone-induced response in SNI rats. These data indicate that the response to acetone in animals with SNI reflects cold allodynia rather than mechanical allodynia.

\section{NPY does not affect behaviour in animals with sham surgery}

To determine whether NPY alters tactile or cold sensitivity, we evaluated the effects of bilateral intra-RVM injection of a high dose of NPY $(10 \mu \mathrm{g})$ in animals with sham surgery. As illustrated in Fig 9, NPY did not alter the behavioral response to innocuous tactile stimuli (Fig 9A-B), a noxious mechanical stimulus (Fig 9C), or a cold stimulus (Fig 9D). By contrast, close inspection of Fig 3D indicates that NPY increased MMF responses in the contralateral paw of nerve-injured rats. This comparison points to the importance of including sham-operated control groups in this type of study.

\section{DISCUSSION}

\section{Medullary facilitation spans multiple models of neuropathic pain}

The past several years have generated an explosion of research directed towards an understanding of the pathophysiological neural changes associated with neuropathic pain. Much of this research stems from the development of new animal models of peripheral nerve injury (Taylor 2001). These models share some important characteristics, such as mechanical allodynia, ectopic firing, and changes in the primary afferent expression of various peptides and neurotransmitter receptors. Our first goal was to determine whether a fundamental finding underlying the concept of medullary descending facilitation, e.g. local anesthetic block of the RVM leading to inhibition of neuropathic pain (Pertovaara et al. 1996; Chen et al. 2004), is restricted to mechanical allodynia associated with the spinal nerve ligation model, or is representative of a more global phenomenon encompassing multiple behavioural signs of hypersensitivity across multiple models of neuropathic pain. Two key observations in our current studies support the latter conclusion. First, microinjection of lidocaine into the RVM reduced mechanical allodynia after either PSNL or SNI, thus extending the previous findings after SNL to alternative models of neuropathic pain. Our second key observation supporting our primary conclusion is that microinjection of lidocaine into the RVM inhibits cold 
hypersensitivity and/or mechanical hyperalgesia, indicating that the RVM relays multimodal descending facilitatory information to the dorsal horn. The magnitude (but not direction) of the lidocaine effect varied between the PSNL and SNI studies and the behavioural endpoints, possibly due to environmental factors -- the PSNL and SNI studies were performed in different laboratories. Alternatively, variations in magnitude could result from the use of different microinjection approaches. In the SNI studies, we utilized a bilateral microinjection approach so as to mimic previous studies and to target the more lateral Y1 receptors illustrated in Fig 1. In the PSNL studies, however, we utilized a microinjection approach to target the midline Y1 receptors emphasized by Hokfelt and colleagues (Kopp et al). Without direct comparison of the two approaches in a single neuropathic pain model, we can only speculate that midline Y1 receptors are more sensitive to the anti-allodynia effects of intracranial NPY.

Substantial evidence supports the concept that descending neurons originating in the RVM contribute to facilitation in the dorsal horn, and therefore these are most likely the target of focal lidocaine (Porreca et al. 2002). For example, neurochemical lesions restricted to cell bodies within the RVM inhibit mechanical allodynia (Porreca et al. 2001). Of course, lidocaine inhibits the activity not only of neuronal cell bodies, but also axonal processes. Thus, we cannot rule out the possibility that axons of passage (rather than resident neurons) are the target of intraparenchymal lidocaine injections into the RVM.

\section{Activation of NPY receptors in the midline RVM inhibits neuropathic pain}

Our second goal was to evaluate the behavioral responses to exogenous administration of NPY in two models of neuropathic pain. Because Hokfelt and colleagues found NPY Y1 receptors in the midline raphe magnus (Kopp et al. 2002), we chose a unilateral injection approach targeted to this region. We found that NPY dose-dependently inhibited the mechanical and cold hypersensitivity associated with SNI or PSNL. Close inspection of the SNI data indicates that the effect of NPY was more robust than lidocaine; this furthers our assertion that NPY exerts a powerful analgesic action at the RVM. We speculate that one reason for the lesser effect of lidocaine is due to its inhibition not only of pro-allodynic pathways but also antinociceptive pathways, while NPY only inhibits the former. To test this idea, future neurophysiological studies could evaluate the effects of NPY on the activity of On and Off cells within the RVM (Fields et al. 1991).

Based on the results of our receptor antagonist studies, we suggest that the microinjection of NPY inhibits behavioural signs of neuropathic pain by acting at Y1 receptors in the RVM. Further evidence for the contribution of Y1 receptors could be provided with future studies describing a shift in the NPY dose-response curve using BIBO3304 in the SNI model. Whether the targeted Y1 receptors are pre- or post-synaptic remains to be determined; studies in inflammatory pain models indicate that $\mathrm{Y} 1$ agonists act predominantly at postsynaptic $\mathrm{Y} 1$ receptors to inhibit neuronal activity (Taiwo and Taylor 2002).

NPY is co-stored with norepinephrine in sympathetic nerves innervating cardiovascular tissue (Ekblad et al. 1984; Hakanson et al. 1986). In the cerebral vasculature, NPY causes constriction of vascular smooth muscle (Edvinsson 1985;Abounader et al. 1995) and potentiates the vasoconstrictor effects of norepinephrine (Ekblad et al. 1984); more recent studies suggest that NPY may also act at the endothelium to produce vasodilation (You et al. 2001). We cannot exclude the possibility that some amount of vasoconstriction may have occurred in our studies, and these considerations raise the possibility that NPY decreased allodynia not by a receptormediated neuronal action, but by local vasoconstriction-induced ischemia and thus injury to nociceptive neurons. We do not believe our results stem from such a toxic effect, since the duration of analgesic effects lasted only $1-2 \mathrm{hr}$, as expected for a fairly short-acting local anesthetic. Such reversibility indicates a pharmacological, rather than a toxicological, mechanism of action involving sodium channels. 
Microinjection of the Y1 receptor antagonist, BIBO3304, by itself had no significant effect on mechanical or thermal responses, arguing against an NPY-mediated ongoing inhibition of neuropathic pain. However, because we only used one dose of a single Y1 antagonist, further studies are required to address the potential contribution of endogenous $\mathrm{Y} 1$ receptor activation to the magnitude of allodynia and hyperalgesia.

In conclusion, medullary facilitation is not merely a phenomenological observation restricted to a single sensory modality within a single model of neuropathic pain, but rather represents a more general phenomenon. Our results greatly strengthen the evidence that the RVM contributes to the descending facilitation of neuropathic pain, and provide new evidence that NPY agonists possibly act at the Y1 receptor to inhibit this pathway. Y1 receptors in the RVM may therefore represent an important new pharmacotherapeutic target for the treatment of neuropathic pain.

\section{Acknowledgements}

The study was supported by NIH grants DA10356 and NS45954 to BKT.

\section{References}

Abounader R, Villemure JG, Hamel E. Characterization of neuropeptide Y (NPY) receptors in human cerebral arteries with selective agonists and the new Y1 antagonist BIBP 3226. Br J Pharmacol 1995;116(4):2245-2250. [PubMed: 8564255]

Chaplan SR, Bach FW, Pogrel JW, Chung JM, Yaksh TL. Quantitative assessment of tactile allodynia in the rat paw. Journal of Neuroscience Methods 1994;53:55-63. [PubMed: 7990513]

Chen Q, King T, Vanderah TW, Ossipov MH, Malan TP Jr, Lai J, Porreca F. Differential blockade of nerve injury-induced thermal and tactile hypersensitivity by systemically administered brainpenetrating and peripherally restricted local anesthetics. J Pain 2004;5(5):281-289. [PubMed: 15219260]

Decosterd I, Allchorne A, Woolf CJ. Differential analgesic sensitivity of two distinct neuropathic pain models. Anesth Analg 2004;99(2):457-463. [PubMed: 15271725]table of contents

Decosterd I, Woolf CJ. Spared nerve injury: an animal model of persistent peripheral neuropathic pain. Pain 2000;87(2):149-158. [PubMed: 10924808]

Edvinsson L. Characterization of the contractile effect of neuropeptide $\mathrm{Y}$ in feline cerebral arteries. Acta Physiol Scand 1985;125(1):33-41. [PubMed: 2864784]

Ekblad E, Edvinsson L, Wahlestedt C, Uddman R, Hakanson R, Sundler F. Neuropeptide Y co-exists and co-operates with noradrenaline in perivascular nerve fibers. Regul Pept 1984;8(3):225-235. [PubMed: 6379758]

Erichsen HK, Hao JX, Xu XJ, Blackburn-Munro G. Comparative actions of the opioid analgesics morphine, methadone and codeine in rat models of peripheral and central neuropathic pain. Pain 2005;116(3):347-358. [PubMed: 15982817]

Fields HL, Heinricher MM, Mason P. Neurotransmitters in nociceptive modulatory circuits. Annu Rev Neurosci 1991;14:219-245. [PubMed: 1674413]

Hakanson R, Wahlestedt C, Ekblad E, Edvinsson L, Sundler F. Neuropeptide Y: coexistence with noradrenaline. Functional implications Prog. Brain Res 1986;68:279-287.

Kim KJ, Yoon YW, Chung JM. Comparison of three rodent neuropathic pain models. Exp Brain Res 1997;113(2):200-206. [PubMed: 9063706]

Kopp J, Xu ZQ, Zhang X, Pedrazzini T, Herzog H, Kresse A, Wong H, Walsh JH, Hokfelt T. Expression of the neuropeptide Y Y1 receptor in the CNS of rat and of wild-type and Y1 receptor knock-out mice. Focus on immunohistochemical localization. Neuroscience 2002;111(3):443-532. [PubMed: 12031341]

Miki K, Zhou QQ, Guo W, Guan Y, Terayama R, Dubner R, Ren K. Changes in gene expression and neuronal phenotype in brain stem pain modulatory circuitry after inflammation. J Neurophysiol 2002;87(2):750-760. [PubMed: 11826044] 
Paxinos, G.; Watson, C. The rat brain in stereotaxic coordinates. Orlando, FL: Academic Press, Inc; 1997.

Pertovaara A, Ostergard M, Anko ML, Lehti-Koivunen S, Brandt A, Hong W, Korpi ER, Panula P. RFamide-related peptides signal through the neuropeptide FF receptor and regulate pain-related responses in the rat. Neuroscience 2005;134(3):1023-1032. [PubMed: 16039797]

Pertovaara A, Wei H, Hamalainen MM. Lidocaine in the rostroventromedial medulla and the periaqueductal gray attenuates allodynia in neuropathic rats. Neurosci Lett 1996;218(2):127-130. [PubMed: 8945744]

Porreca F, Burgess SE, Gardell LR, Vanderah TW, Malan TP Jr, Ossipov MH, Lappi DA, Lai J. Inhibition of neuropathic pain by selective ablation of brainstem medullary cells expressing the mu-opioid receptor. J Neurosci 2001;21(14):5281-5288. [PubMed: 11438603]

Porreca F, Ossipov MH, Gebhart GF. Chronic pain and medullary descending facilitation. Trends Neurosci 2002;25(6):319-325. [PubMed: 12086751]

Ren K, Dubner R. Descending modulation in persistent pain: an update. Pain 2002;100(1-2):1-6. [PubMed: 12435453]

Robinson D, Calejesan AA, Zhuo M. Long-lasting changes in rostral ventral medulla neuronal activity after inflammation. J Pain 2002;3(4):292-300. [PubMed: 14622753]

Seltzer Z, Dubner R, Shir Y. A novel behavioral model of neuropathic pain disorders produced in rats by partial sciatic nerve injury. Pain 1990;43(2):205-218. [PubMed: 1982347]

Taiwo OB, Taylor BK. Antihyperalgesic effects of intrathecal neuropeptide Y during inflammation are mediated by Y1 receptors. Pain 2002;96(3):353-363. [PubMed: 11973010]

Taylor BK. Pathophysiologic mechanisms of neuropathic pain. Curr Pain Headache Rep 2001;5(2):151161. [PubMed: 11252149]

Taylor BK, Basbaum AI. Neurochemical characterization of extracellular serotonin in the rostral ventromedial medulla and its modulation by noxious stimuli. J Neurochem 1995;65(2):578-589. [PubMed: 7616212]

Taylor BK, Basbaum AI. Systemic morphine-induced release of serotonin in the rostroventral medulla is not mimicked by morphine microinjection into the periaqueductal gray. J Neurochem $2003 ; 86(5)$ : 1129-1141. [PubMed: 12911621]

Wolak ML, DeJoseph MR, Cator AD, Mokashi AS, Brownfield MS, Urban JH. Comparative distribution of neuropeptide Y Y1 and Y5 receptors in the rat brain by using immunohistochemistry. J Comp Neurol 2003;464(3):285-311. [PubMed: 12900925]

You J, Edvinsson L, Bryan RM Jr. Neuropeptide Y-mediated constriction and dilation in rat middle cerebral arteries. J Cereb Blood Flow Metab 2001;21(1):77-84. [PubMed: 11149671]

Zhang Y, Lundeberg T, Yu L. Involvement of neuropeptide Y and Y1 receptor in antinociception in nucleus raphe magnus of rats. Regul Pept 2000;95(1-3):109-113. [PubMed: 11062340] 

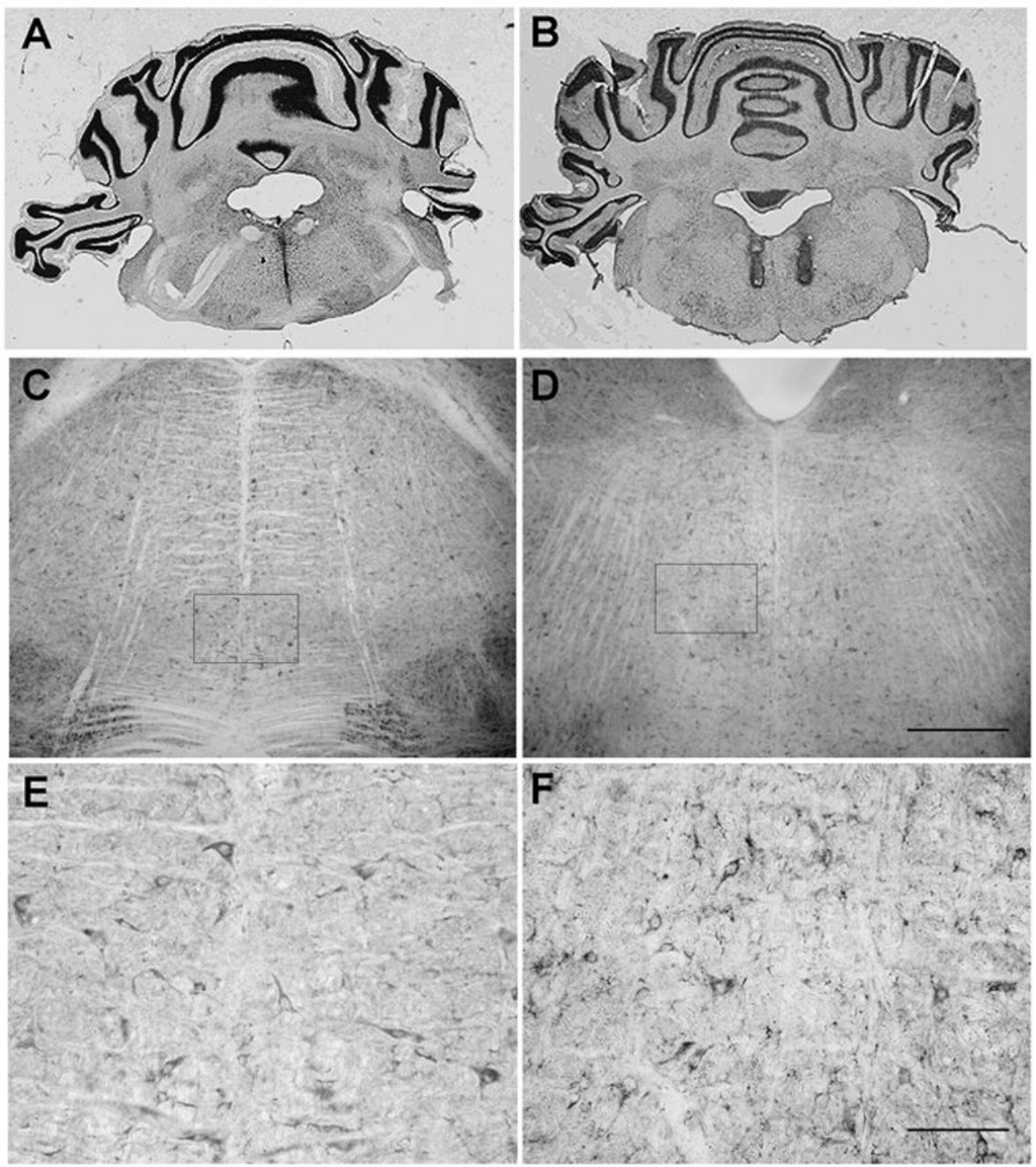

Figure 1. Microinjection sites within the RVM

Photomicrographs showing placement of A) midline single cannula tract (bregma - $10.04 \mathrm{~mm}$ ) and B) bilateral cannula tracts (bregma $-10.80 \mathrm{~mm}$ ) within the RVM in brain sections counterstained with cresyl violet. Section thickness $=40 \mu \mathrm{m}$. Brightfield photomicrographs of $\mathrm{Y} 1$ receptor immunoreactivity in the RVM at C) bregma $-10.08 \mathrm{~mm}$ and $\mathrm{D}$ ) bregma -10.80 $\mathrm{mm}$. Scale bars $=200 \mu \mathrm{m}$ for $\mathrm{C}$, D. The boxed areas in C and D represent areas of higher magnification as shown in E and F, respectively. Scale bars $=100 \mu \mathrm{m}$ for E, F. 

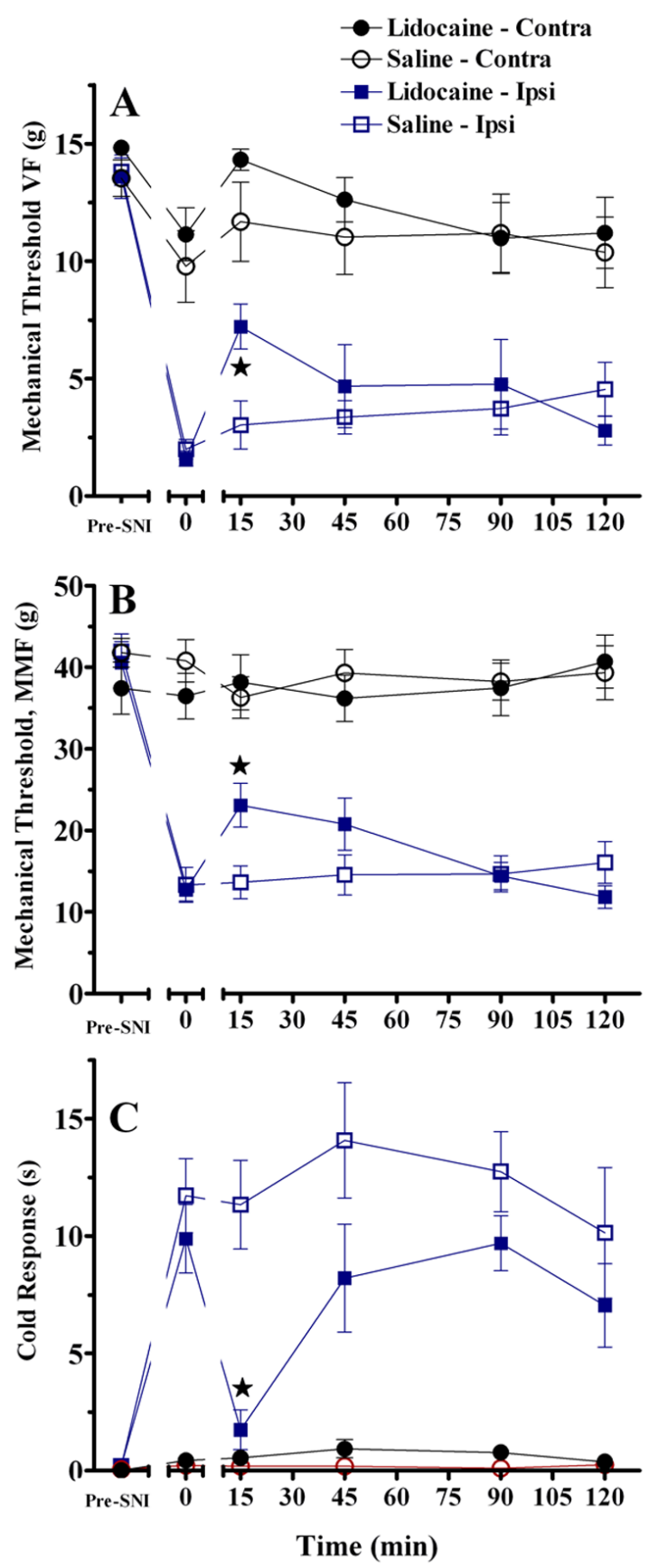

Figure 2. Intra-RVM lidocaine decreases spared nerve injury (SNI)-induced allodynia Bilateral microinjection of $2 \%$ lidocaine (A-C) or unilateral microinjection of $4 \%$ lidocaine (D-F) into the RVM. Lidocaine reversed the drop in mechanical threshold as assessed with von Frey hairs [A,D, $\mathrm{P}<0.01]$, and a machine-mounted filament $(\mathbf{B}, \mathrm{MMF}, \mathrm{P}<0.01)$. Lidocaine also reversed cold allodynia $(\mathbf{C , F})$ assessed as the duration of response to a drop of acetone. Lidocaine did not change responses at the paw contralateral to SNI. $n=6-15$. Error bars $=$ SEM. $\star \mathrm{P}<0.01$, saline vs lidocaine at the ipsilateral hindpaw, Mann-Whitney U test subsequent to Kruskal Wallis (A) or post-hoc Bonferroni subsequent to ANOVA (B-C). 

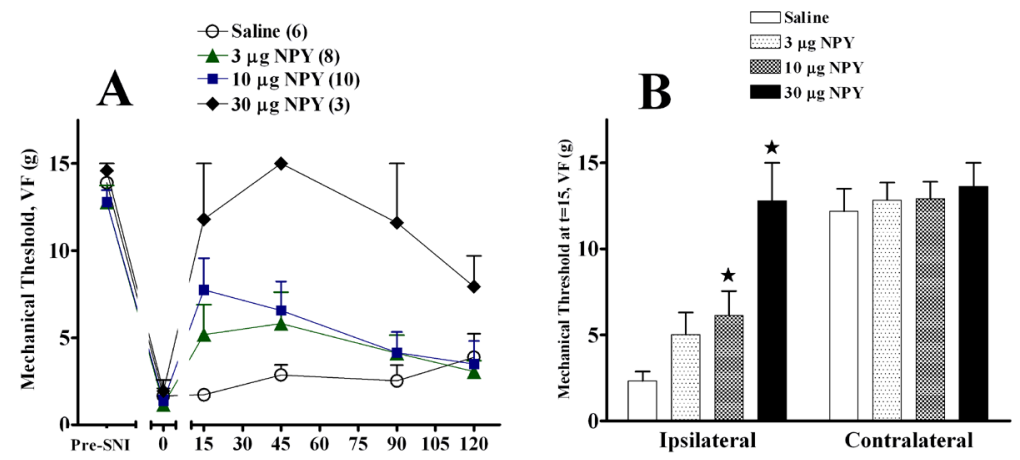

C

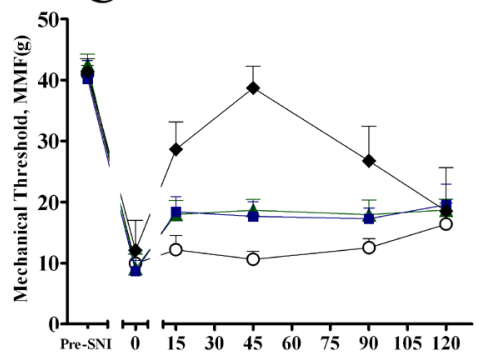

D
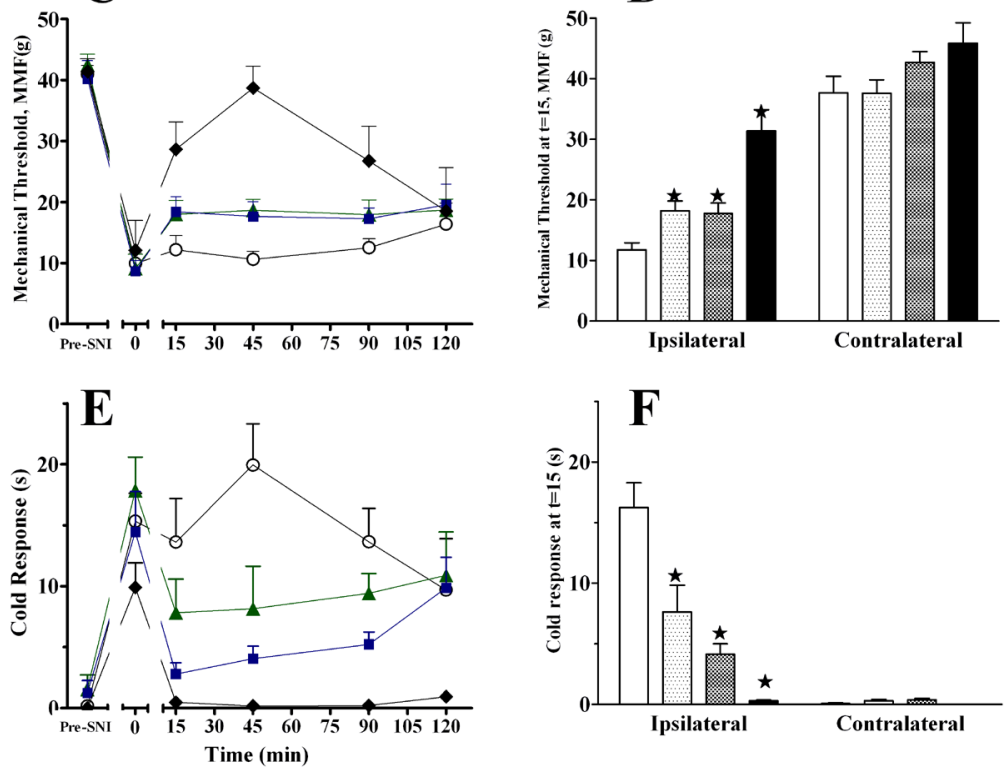

Figure 3. Intra-RVM NPY decreases SNI -induced allodynia

Unilateral microinjection of NPY into the midline RVM dose-dependently reduced mechanical allodynia (von Frey, Panels A-B; machine-mounted filament, Panels C-D), and cold allodynia (Panel E-F). The line graphs in panels A, C, E illustrate time course data collected from the paw ipsilateral to SNI. The histograms in panels B, D, F summarize the data obtained at the $15^{\prime}$ time point from the ipsilateral and contralateral sides. Numbers of animals in each group are in parenthesis. Error bars $=$ SEM. $\star$ P $<0.05$ vs saline, Mann-Whitney U test subsequent to Kruskal Wallis (A-B) or post-hoc Bonferroni subsequent to ANOVA $(\mathrm{C}-\mathrm{F})$. 

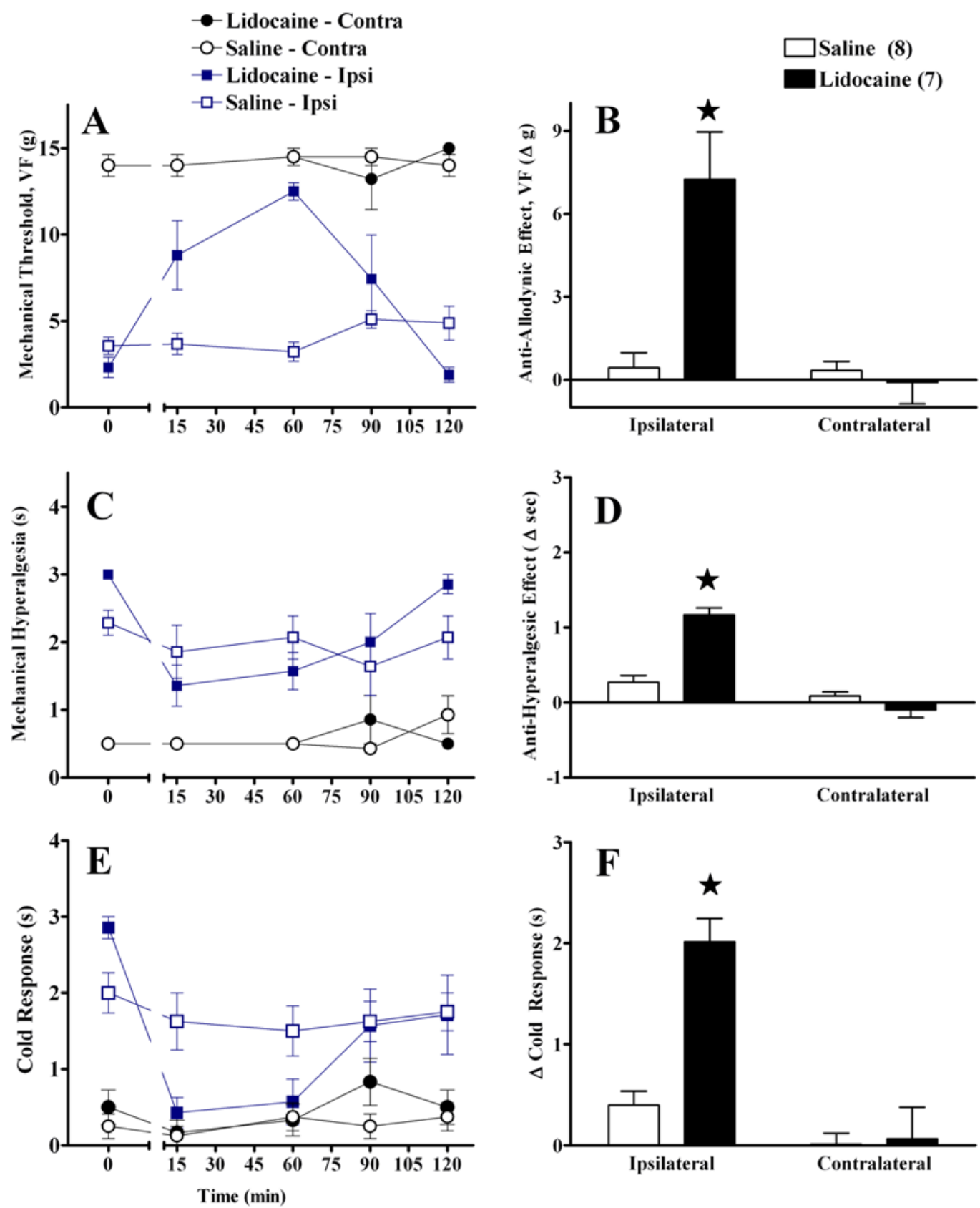

Figure 4. Intra-RVM lidocaine decreases partial sciatic ligation (PSNL)-induced allodynia and hyperalgesia

Unilateral microinjection of lidocaine into the midline RVM reduced mechanical allodynia (VF, Panels A-B), mechanical hyperalgesia (Panels C-D), and cold allodynia (Panel E-F). The line graphs in panels A, C, E illustrate time course data. The histograms in panels B, D, F summarize the change in behavior from baseline $(t=0)$ to post injection (mean of $t=15-90)$ at the ipsilateral and contralateral sides. Lidocaine did not changes responses at the paw contralateral to PSNL. Numbers of animals in each group are in parenthesis. Error bars = SEM. $\star \mathrm{P}<0.05$ vs saline, Mann-Whitney U test subsequent to Kruskal Wallis (A-B) or post-hoc Bonferroni subsequent to repeated-measures ANOVA $(\mathrm{C}-\mathrm{F})$. 

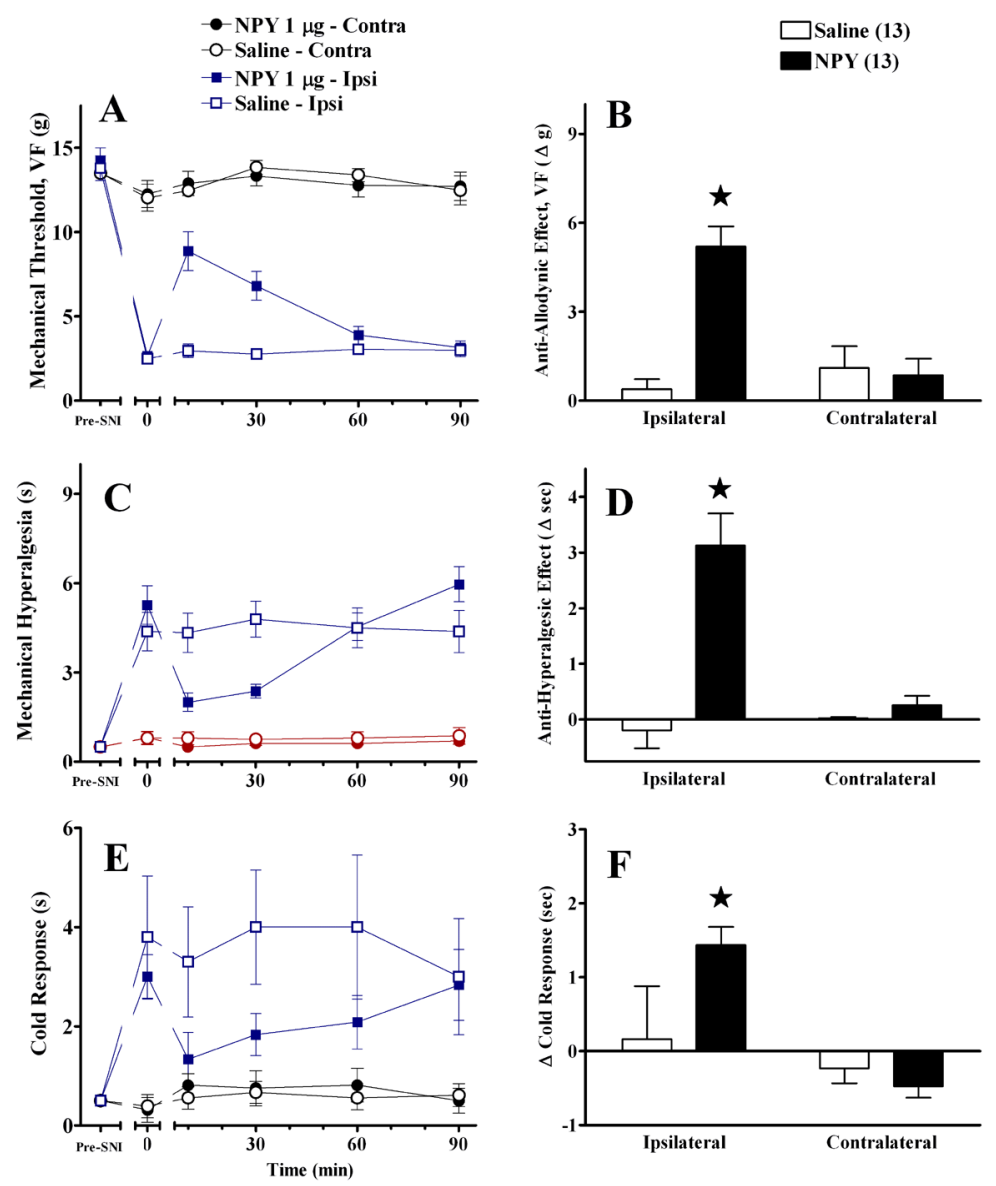

Fig 5. Intra-RVM NPY decreases PSNL -induced allodynia and hyperalgesia

Unilateral microinjection of NPY into the midline RVM reduced mechanical allodynia (Panels A-B) mechanical hyperalgesia (Panels C-D), and cold allodynia (Panel E-F). The line graphs in panels A, C, E illustrate time course data collected from the paw ipsilateral to SNI. The histograms in panels $\mathrm{B}, \mathrm{D}, \mathrm{F}$ summarize the change in behavior from baseline $(\mathrm{t}=0)$ to post injection (mean of $\mathrm{t}=10-30$ ) at the ipsilateral and contralateral sides. NPY did not changes responses at the paw contralateral to SNI. Numbers of animals in each group are in parenthesis. Error bars = SEM. $\star \mathrm{P}<0.05$ vs saline, Mann-Whitney U test subsequent to Kruskal Wallis (A-B) or post-hoc Bonferroni subsequent to repeated-measures ANOVA (C-F). 

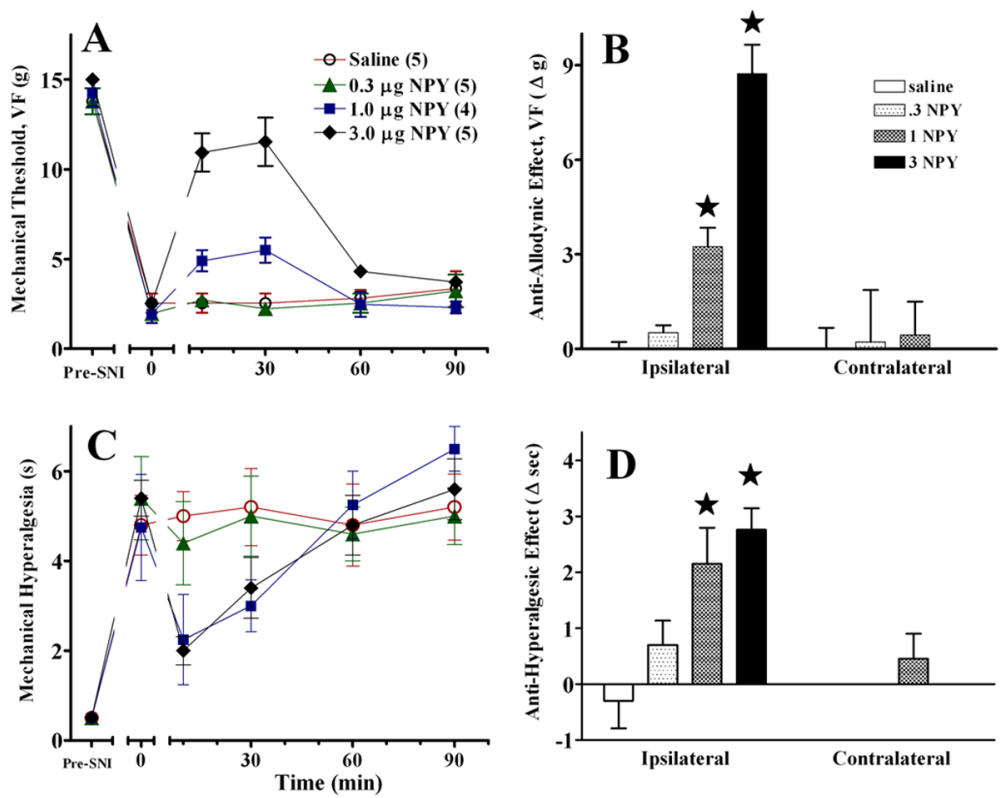

Figure 6. Intra-RVM NPY dose-dependently decreases PSNL-induced allodynia and hyperalgesia The line graphs in panels A and C illustrate time course data collected from the paw ipsilateral to SNI. The histograms in panels B and D summarize the change in von Frey threshold from baseline $(\mathrm{t}=0)$ to post injection (mean of timepoints 10-30) at the ipsilateral and contralateral sides. Error bars = SEM. Numbers of animals in each group are in parenthesis. $\star \mathrm{P}<0.05$, Mann-Whitney U test subsequent to Kruskal Wallis (A-B) or post-hoc Bonferroni subsequent to repeated-measures ANOVA (C-D). 

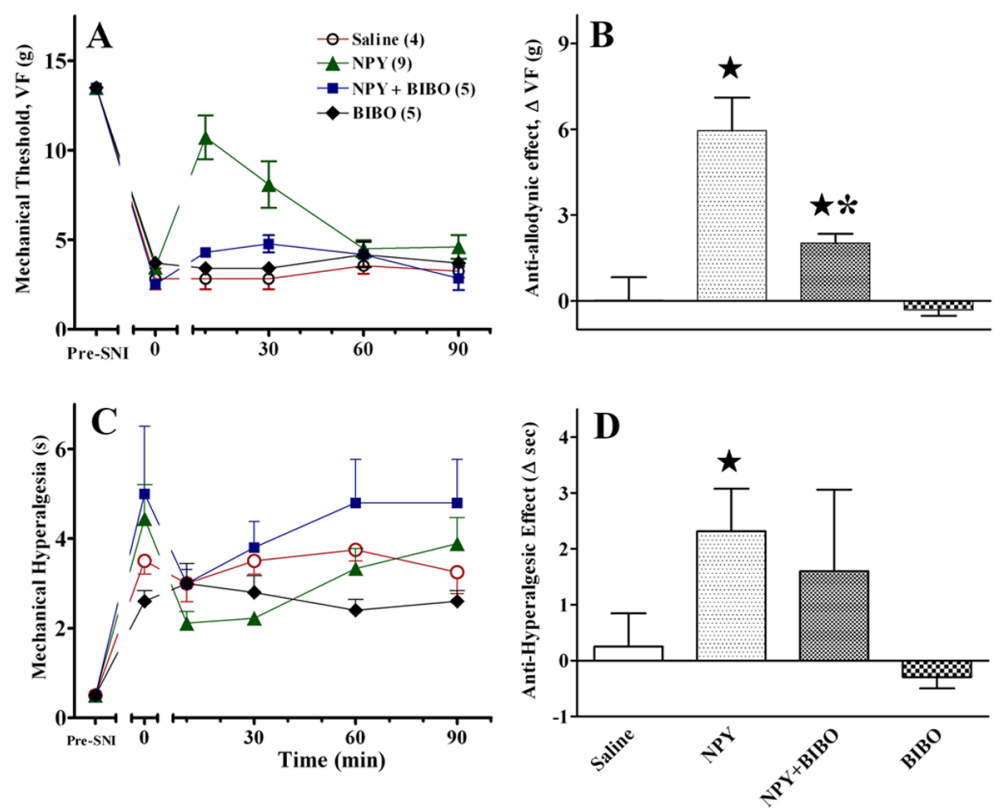

Figure 7. Intra-RVM delivery of the selective Y1 receptor antagonist BIBO3304 reverses the effects of NPY in the PSNL model

When administered 15 min before NPY $(1 \mu \mathrm{g})$, the unilateral microinjection BIBO $(1 \mu \mathrm{g})$ but not saline prevented the anti-allodynic (Panels A-B) and anti-hyperalgesic (Panels C-D) effects of NPY at the hindpaw ipsilateral to nerve injury. BIBO had no effect when administered alone. Numbers of animals in each group are in parenthesis. Error bars $=$ SEM. $\star \mathrm{P}<0.05$ vs saline, ${ }^{*} \mathrm{P}<0.05$ vs NPY alone, Mann-Whitney U test subsequent to Kruskal Wallis or posthoc Bonferroni subsequent to ANOVA. 

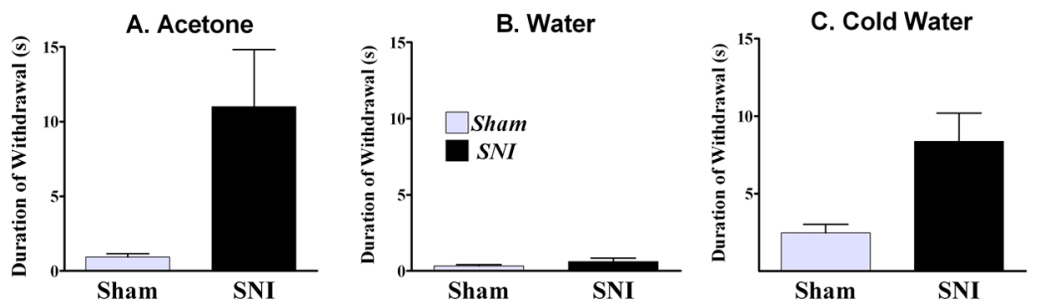

Figure 8. The acetone test is a behavioral measure of cold hypersensitivity

Time spent lifting the ipsilateral paw over a 60-s observation period in response to a drop of acetone (A), water (B), or to its immersion in 1-cm deep ice water (C) after sham ( $\mathrm{n}=3$ ) or SNI surgery $(n=7)$. In contrast to acetone, a drop of water did not evoke a robust response. However, ice water produced a greater response in SNI rats as compared to Sham rats; no difference was observed at the contralateral paw. These data indicate that the response to acetone reflects cold hypersensitivity rather than mechanical allodynia. Error bars = SEM. 


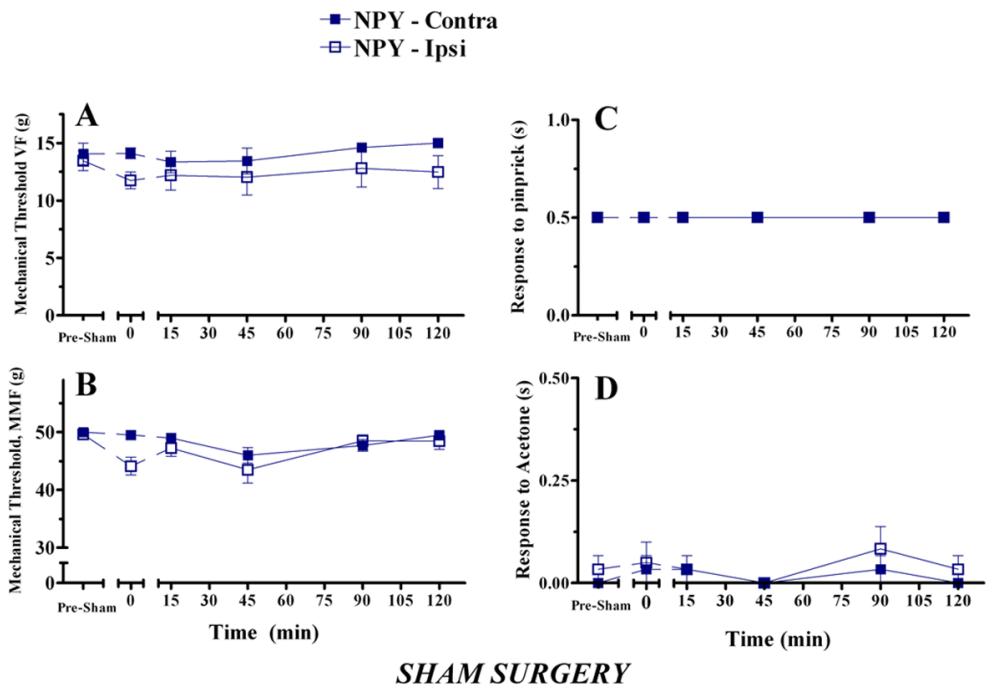

Figure 9. Intra-RVM delivery of NPY does not affect behaviour in animals with sham surgery Behavior was evaluated in the ipsilateral and contralateral paws (relative to sham surgery) before and after microinjection of NPY ( $5 \mu \mathrm{g}$ bilateral) into the RVM at $\mathrm{t}=0$. NPY did not change the withdrawal response to innocuous mechanical stimuli (Panels A-B), a noxious mechanical stimulus (Panel C), or a cold stimulus (Panel D). $\mathrm{n}=6$. 